\title{
Can a changing $\alpha$ explain the Supernovae results?
}

\author{
John D. Barrow ${ }^{1}$ and João Magueijo ${ }^{2}$ \\ ${ }^{1}$ DAMTP, Cambridge University, Silver St., Cambridge CB3 9EW, U.K. \\ ${ }^{2}$ Theoretical Physics Group, The Blackett Laboratory, \\ Imperial College, Prince Consort Rd., London, SW7 2BZ, U.K.
}

Received _ _ 


\begin{abstract}
We show that the Supernovae results, implying evidence for an accelerating Universe, may be closely related to the recent discovery of redshift dependence in the fine structure constant $\alpha$. The link is a class of varying speed of light (VSL) theories which contain cosmological solutions similar to quintessence. During the radiation dominated epoch the cosmological constant $\Lambda$ is prevented from dominating the Universe by the usual VSL mechanism. In the matter epoch the varying $c$ effects switch off, allowing $\Lambda$ to eventually surface and lead to an accelerating Universe. By the time this happens the residual variations in $c$ imply a changing $\alpha$ at a rate that is in agreement with observations.
\end{abstract}

Subject headings: Cosmology: theory - observation - Hubble diagram, cosmological constant, high redshift spectroscopy 


\section{Introduction}

Two puzzling observations are challenging cosmologists. The Supernovae project (Perlmutter et al 97; see also Garnavich et al 98, Schmidt 98, Riess et al 98) has extended the reach of the Hubble diagram to high redshift and provided new evidence that the expansion of the universe is accelerating. This implies that there exists a significant positive cosmological constant, $\Lambda$. In separate work, the spacings between quasar absorption lines were examined in Keck I data at medium redshifts, $z \sim 1$, (Webb et al 99) and compared with those in the laboratory (see also Drinkwater et al 98, Damour and Dyson 96, Shylakhter 76, Barrow 87). These observations are sensitive to time variations in the value of the fine structure constant $\alpha=e^{2} /(\hbar c)$, (where $e$ is the electron charge, $\hbar$ Planck's constant, and $c$ the speed of light), at a rate one million times slower that the expansion rate of the universe. This is a more sensitive cosmological probe than COBE anisotropy measurements of the microwave background. These observations found evidence for a small variation in the value of $\alpha$ at redshifts $\sim 1$. This could be produced by intrinsic time variation or by some unidentified line-blending effect. In this Letter we assume that the variation is intrinsic and show that there may be a link between the observations of cosmological acceleration and varying $\alpha$.

If $\Lambda>0$, then cosmology faces a very serious fine tuning problem, and this has motivated extensive theoretical work (Zlatev et al 99). There is no theoretical motivation for a value of $\Lambda$

of currently observable magnitude; a value $10^{120}$ times smaller that the 'natural' Planck scale of density is needed if $\Lambda$ becomes important near the present time. Such a small non-zero value of $\Lambda$ is 'unnatural' in the sense that making it zero reduces the symmetry of spacetime. A possible solution is quintessence (Zlatev et al 99): the idea that $\Lambda$ might be a rolling scalar field exhibiting very long transients. Here we introduce another explanation.

There are a variety of possible physical expressions of a changing $\alpha$. Bekenstein proposed a varying $e$ theory (Bekenstein 82). An alternative is the varying speed of light (VSL) theory (Moffat 93, Albrecht \& Magueijo 99, Barrow 99) in which varying $\alpha$ is expressed as a variation of the speed of light. The choice between these two types of theory transcends experiment, and merely 
reflects theoretical convenience in the choice of units (Barrow \& Magueijo 98). The simplest cosmology following from VSL is known to contain an attractor in which $\Lambda$ and matter remain at fixed density ratios throughout the life of the universe (Barrow \& Magueijo 99). Such attractor solves the fine tuning problem forced upon us by the supernovae results. Hence there is scope for the observed changing $\alpha$ to be related to the observed acceleration of the universe. In this Letter we propose a theory which leads to good quantitative agreement, given experimental errors, between the observations of acceleration and varying $\alpha$. In Section 2 we examine the construction of the Hubble diagram in VSL theories, and the interpretation of varying- $\alpha$ experiments. Then in Section 3 we present an example of a VSL model which can jointly explain the supernovae results and the Webb et al varying- $\alpha$ results. We conclude with a discussion of some further aspects of the model proposed, to be investigated elsewhere.

\section{The VSL Hubble diagram}

The Hubble diagram is a plot of luminosity distance against redshift. The purpose is to map the expansion factor $a(t)$, where $t$ is the comoving proper time. Redshifts provide a measurement of $a$ at the time of emission. If the objects under observation are "standard candles" (as Type Ia supernovae are assumed to be), their apparent brightness gives their (luminosity) distance, which, if we know $c$, tells us their age. By looking at progressively more distant objects we can therefore map the curve $a(t)$.

We now examine how this construction is affected by a changing $c$. In Albrecht \& Magueijo 99 we showed that $E \propto c^{2}$ for photons in free flight. We also showed that quantum mechanics remains unaffected by a changing $c$ if $\hbar \propto c$ (in the sense that quantum numbers are adiabatic invariants). If $\hbar \propto c$, the Rydberg energy scales like $c^{2}$ :

$$
E_{R}=\frac{m_{e} c^{4} e^{4}}{2 \hbar^{2}} \propto c^{2}
$$

Hence all absorption lines, ignoring the fine structure, scale like $c^{2}$. When we compare lines from 
near and far systems we should therefore see no effects due to a varying $c$; the redshift $z$ is still

$$
1+z_{e}=a_{o} / a_{e}
$$

where $o$ and $e$ label epochs of observation and emission.

In order to examine luminosity distances, we need to reassess the concept of standard candles. For simplicity let us first treat them as black bodies. Then their temperature scales as $T \propto c^{2}$ (Albrecht \& Magueijo 99), their energy density scales as $\rho \propto T^{4} /(\hbar c)^{3} \propto c^{2}$, and their emission power as $P=\rho / c \propto c$, implying that standard candles are brighter in the early universe if $\dot{c}<0$. However, the power emitted by these candles, in free flight, scales like $c$; each photon's energy scales like $c^{2}$, its speed like $c$, and therefore its energy flux like $c$. The received flux, as a function of $c$, therefore scales like:

$$
P_{r}=\frac{P_{e} c^{2}}{4 \pi r^{2} c} \propto c
$$

where $r$ is the conformal distance to the emitting object, and the subscripts $r$ and $e$ label received and emitted. In an expanding universe we therefore still have

$$
P_{r}=\frac{P_{e 0}}{4 \pi r^{2} a_{0}^{2}}\left(\frac{a}{a_{o}}\right)^{2}
$$

where $P_{e 0}$ is the emitting power of standard candles today. Notice that the above argument is still valid if the candles are not black bodies; it depends only on the scaling properties of emitted and received power.

We can now set up the Hubble diagram. Consider the Taylor expansion

$$
a(t)=a_{0}\left[1+H_{0}\left(t-t_{0}\right)-\frac{1}{2} q_{0} H_{0}^{2}\left(t-t_{0}\right)^{2}+\ldots\right]
$$

where $H_{0}=\dot{a}_{0} / a_{0}$ is the Hubble constant, and $q_{0}=-\ddot{a}_{0} a_{0} / \dot{a}_{0}^{2}$ is the decceleration parameter. Hence

$$
z=H_{0}\left(t_{0}-t\right)+\left(1+q_{0} / 2\right) H_{0}^{2}\left(t-t_{0}\right)^{2}+\ldots .
$$

or

$$
t_{0}-t=\frac{1}{H_{0}}\left[z-\left(1+q_{0} / 2\right) z^{2}+\ldots\right] .
$$


From (雨) we find that the luminosity distance $d_{L}$ is

$$
d_{L}=\left(\frac{P_{e 0}}{4 \pi P_{0}}\right)^{1 / 2}=a_{0}^{2} \frac{r}{a}=a_{0} r\left(1+z_{e}\right) .
$$

The conformal distance to the emitting object is given by

$$
r=\int_{t}^{t_{0}} \frac{c(t) d t}{a(t)}
$$

From (5) we have that

$$
r=c_{0}\left[\left(t_{0}-t\right)+\frac{1-n}{2} H_{0}\left(t_{0}-t\right)^{2}+\ldots\right]
$$

where we have assumed that locally $c=c_{0} a^{n}$ (that is $c=c_{0}\left[1+n H_{0}\left(t-t_{0}\right)+\ldots\right]$ ). Substituting (7) we finally have :

$$
d_{L}=\frac{c_{0}}{H_{0}}\left[z+\frac{1}{2}\left(1-\left(q_{0}+n\right)\right) z^{2}+\ldots\right]
$$

We see that besides the direct effects of VSL upon the expansion rate of the universe, it also induces an effective acceleration in the Hubble diagram as an "optical illusion" (we are assuming that $c$ decreases in time: $n<0)$. This is easy to understand. We have seen that VSL introduces no intrinsic effects in the redshifting spectral line or in the dimming of standard candles with distance and expansion. The only effect VSL induces on the construction of the Hubble diagram is that for the same redshift (that is, the same distance into the past) objects are farther away from us because light travelled faster in the past. But an excess luminosity distance, for the same redshift, is precisely the hallmark of cosmological acceleration. However, we need to consider the other experimental input to our work: the Webb et al 99 results. By measuring the fine structure in absorption systems at redshifts $z \sim O(1)$ we can also map the curve $c(t)$. Since $c=c_{0}\left[1+n H_{0}\left(t-t_{0}\right)+\ldots\right]$ we have $c=c_{0}[1-n z+\ldots]$, and so to first order $\alpha=\alpha_{0}[1+2 n z+\ldots]$. However, the results presented in Webb et al 99 show that $n$ is at most of order $10^{-5}$. This means that the direct effects of varying $c$ permitted by the quasar absorption system observations are far too small to explain the observed acceleration. We need to look at a fully self-consistent generalisation of general relativity containing the scope for varying $c$. 


\section{The model}

We start with some general properties of the dynamics of $c$ (see Barrow \& Magueijo 99b for further discussion). Drawing inspiration from dilaton theories (like Brans-Dicke gravity) we take

$$
\psi=\log \left(c / c_{0}\right)
$$

as the dynamical field associated with $c$. Indeed, powers of $c$ appear in all coupling constants, which in turn can be written as $e^{\phi}$, where $\phi$ is the dilaton. We then endow $\psi$ with a dynamics similar to the dilaton. The left-hand side for the $\psi$ equation for a homogeneous field in an expanding universe should be $\ddot{\psi}+3 \frac{\dot{a}}{a} \dot{\psi}$. This structure ensures that the propagation equation for $\psi$ is second-order and hyperbolic. However, since VSL breaks Lorentz invariance one need not choose (as in Brans-Dicke) the source term to be $\rho-3 p$, where $\rho$ and $p$ are the energy density and pressure of matter respectively. Instead, we choose

$$
\ddot{\psi}+3 \frac{\dot{a}}{a} \dot{\psi}=4 \pi G \omega \frac{p}{c^{2}},
$$

where $p$ is the total pressure of the matter fields and $\omega$ is a coupling constant (distinct from the Brans Dicke coupling constant). The full self-consistent system of equations in a matter-plus-radiation universe containing a cosmological constant stress

$$
\rho_{\Lambda}=\frac{\Lambda c^{2}}{8 \pi G}
$$

is therefore

$$
\begin{aligned}
\ddot{\psi}+3 \frac{\dot{a}}{a} \dot{\psi} & =4 \pi G \omega \frac{\rho_{\gamma}}{3}, \\
\dot{\rho}_{\gamma}+4 \frac{\dot{a}}{a} \rho_{\gamma} & =-2 \rho_{\Lambda} \dot{\psi}, \\
\dot{\rho}_{\Lambda} & =2 \rho_{\Lambda} \dot{\psi}, \\
\dot{\rho}_{m}+3 \frac{\dot{a}}{a} \rho_{m} & =0, \\
\left(\frac{\dot{a}}{a}\right)^{2} & =\frac{8 \pi G}{3}\left(\rho_{m}+\rho_{\gamma}+\rho_{\Lambda}\right),
\end{aligned}
$$

where subscripts $\gamma$ and $m$ denote radiation and matter respectively. We have assumed that the sink term (15) is reflected in a source term in (14) (and not in (16)). This is due to the fact that this 
term is only significant very early on, when even massive particles behave like radiation. We have ignored curvature terms because in the quasi-lambda dominated solutions we are about to explore we know that these are smaller than $\rho_{\Lambda}$, (Barrow \& Magueijo 99). Here, in complete contrast to Brans-Dicke theory, the field $\psi$ is only driven by radiation pressure in the dust-dominated era. In other words, only conformally invariant forms of matter couple to the field $\psi$.

In a radiation-dominated universe the behaviour of this system changes at the critical value $\omega=-4$. For $\omega<-4$ we reach a flat $\Lambda=0$ attractor as $t \rightarrow \infty$. For $-4<\omega<0$ we have attractors for which $\rho_{\Lambda}$ and $\rho_{\gamma}$ maintain a constant ratio (see Barrow \& Magueijo 99). In Fig. 1 we plot a numerical solution to this system, with $\omega=-4.4$ (a $10 \%$ tuning below the critical value $\omega=-4$ ) and $n=-2.2$ during the radiation epoch. As expected from Barrow \& Magueijo 99, this forces $\Omega_{\Lambda}$ to drop to zero, while the expansion factor acquires a radiation-dominated form, with $a \propto t^{1 / 2}$. By the time the matter-dominated epoch is reached, $\Omega_{\Lambda}$ is of order $10^{-12}$. During the matter epoch, the source term for $\psi$ disappears in eq. (12), $n$ starts to approach zero, $\Omega_{\Lambda}$ starts to increase, and the expansion factor takes on the $a \propto t^{2 / 3}$ dependence of a matter-dominated universe. A few expansion times into the matter epoch, $\Omega_{\Lambda}$ becomes of order 1 and the universe begins accelerating. By the time this happens $n$ is of order $10^{-5}$, in agreement with the expectations of Webb et al 99. This type of behaviour can be achieved generically, for different initial conditions, with a tuning of $\omega$ that never needs to be finer than a few percent.

We can provide an approximate argument explaining why this theory should display this type of behaviour and why we need so little fine tuning of $\omega$ to explain the supernovae experiments. If we neglect changes in $c$ after matter-radiation equality, $t_{e q}$, we are going to require

$$
\frac{\rho_{\Lambda}\left(t_{e q}\right)}{\rho\left(t_{e q}\right)} \approx z_{e q}^{-3} \sim 10^{-12}
$$

Let $c=c_{0} a^{n(t)}$, with $n=-2-\delta$, and $n=\omega / 2$ during the radiation epoch. We can integrate the conservation equations to give

$$
\frac{\rho}{\rho_{\Lambda}}=\frac{A}{a^{4} \rho_{\Lambda}}-\frac{n}{n+2}
$$


with $A$ constant, from which it follows that

$$
\frac{\rho}{\rho_{\Lambda}}=\frac{2}{\delta}\left[\left(1+\frac{\delta}{2} \frac{\rho_{i}}{\rho_{\Lambda i}}\right)\left(\frac{a}{a_{i}}\right)^{2 \delta}-1\right] .
$$

We see that assymptotically $\rho / \rho_{\Lambda}$ grows to infinity, if $\delta>0$ (the flat $\Lambda=0$ attractor of Barrow \& Magueijo 99). However the growth is very slow even if $\delta$ is not very small. Our theory displays very long transients, and a very slow convergence to its attractor, a property similar to quintessence models (Zlatev et al 99). It is therefore possible to achieve $\rho_{\Lambda} / \rho \sim 10^{-12}$ at the end of the radiation epoch, with $\delta$ chosen to be of order 0.1 .

Now, why is the change in $c$ of the right order of magnitude to explain the results of Webb et al 99? With a solution of the form $c=c_{0} a^{n(t)}$ we find that

$$
n(t) \approx \frac{\omega \rho_{\gamma}}{3\left(\rho_{m}+2 \rho_{\Lambda}\right)}
$$

With $\omega \approx-4$ we therefore have

$$
n\left(t_{0}\right) \approx-\frac{4}{3} \frac{2.3 \times 10^{-5}}{h^{2}\left(1+\Omega_{\Lambda}\right)}
$$

of the right order of magnitude. The order of magnitude of the index $n \sim 10^{-5}$, observed by Webb et al 99, is therefore fixed by the ratio of the radiation and the matter energy densities today.

\section{Discussion}

In this Letter we proposed a theory relating the supernovae results and the observations by Webb et al 99(we refer the reader to Ellis et al 99, Amelino-Camelia et al 98 and Amendola et al 99 for work related to ours). We have shown that a varying $c$ of the same magnitude required to create an apparent change in the fine structure constant at $z \sim 1$ at the observed level is also sufficient to produce an acceleration in the Hubble expansion of the sort inferred from the supernova data. The theory we have proposed is one example within a class whose members exhibit similar behaviour. In these theories the gravitational effect of the pressure drives changes in $c$, and these convert the energy density in $\Lambda$ into radiation. Thus $\Lambda$ is prevented from dominating the universe during the radiation epoch. As the universe cools down, massive particles eventually 
become the source of pressureless matter and create a matter-dominated epoch. In the matter epoch the variation in $c$ comes to a halt, with residual effects at $z \approx 1-5$ at the level observed by Webb et al. As the $c$ variation is switched off, the $\Lambda$ stress resurfaces, and dominates the universe for a few expansion times in the matter-dominated era, in agreement with the supernovae results.

Our scenario has other interesting aspects, beyond the scope of this Letter, which we summarize here. As we go back in time we find that $t / t_{P}$ (where $t_{P}=\left(G h / c^{5}\right)^{1 / 2}$ is the Planck time) increases. Hence the universe does not have a quantum origin, and one need not invoke quantum cosmology to set its initial conditions. In addition to the solution to Eqns. (13)-(17) described in this Letter (which we label a VSL Big Bang solution) there is a trivial stable attractor: a non-VSL de Sitter universe. Even though this solution is stable there is a minimal

perturbation in $\rho_{\gamma}$ or $\dot{\psi}$ (not necessarily over a large volume) which takes it into the Big Bang solution. This minimal perturbation should therefore be regarded as a "natural initial condition" for the Big Bang solution. Such a configuration, the minimal point interpolating two stable solutions, is reminiscent of sphalarons in field theory. Hence a VSL Big Bang solution has a classical de Sitter beginning, and given that it will end in $\Lambda$ domination, the universe as a whole is de Sitter with sporadic Big Bang "events". At the end of a Big Bang phase $\Lambda$ dominates and $c$ stops changing. Then a new fluctuation triggers a VSL Big Bang event. In every Big Bang cycle $c$ drops and $\Lambda$, measured in fundamental units, increases. Its current small value merely measures the number of cycles before ours, starting from an arbitrarily small value. Therefore our scenario not only explains why $\Lambda$ is dominating only now, but also the smallness of $\Lambda$ in fundamental units. Moreover, after a sufficient number of Big Bang cycles, the value of $\Lambda$ in fundamental units will grow to be of order 1 . Thus, quantum cosmology is in the future, not in the past.

\section{Acknowledgements}

JDB is supported by a PPARC Senior Fellowship and JM by the Royal Society. JM would like to thank K. Baskerville and D. Sington for help with this project. 


\section{REFERENCES}

A. Albrecht and J. Magueijo, Phys. Rev. D59 043516 (1999).

G. Amelino-Camelia et al, astro-ph/9810483.

L. Amendola, S. Corasaniti, F. Occhionero, astro-ph/9907222

J.D. Barrow, Phys. Rev. D 35, 1805 (1987).

J.D. Barrow, Phys. Rev. D59 043515 (1999).

J.D. Barrow and J. Magueijo, Phys. Lett. B443 (1998) 104.

J.D. Barrow and J. Magueijo, Phys. Lett. B447 (1999) 246.

J.D. Barrow and J. Magueijo, "A dynamical varying speed of light theory", to be submitted.

J.D. Bekenstein, Phys. Rev. D 25, 1527 (1982)

T. Damour and F. Dyson, Nucl. Phys. B 480, 37 (1996).

M.J. Drinkwater, J.K. Webb, J.D. Barrow \& V.V. Flambaum, Mon. Not. R. astron. Soc. 295, 457 (1998).

J. Ellis, N. E. Mavromatos, D. V. Nanopoulos, gr-qc/9904068.

Garnavich, P.M et al 1998 Ap.J. Letters 493:L53-57.

J.W. Moffat, Int. J. Mod. Phys. D2 (1993) 351.

S. Perlmutter et al, Ap. J. 483, 565 (1997); S. Perlmutter et al (The Supernova Cosmology project), Nature 39151 (1998).

Riess, A.G. et al 1998 Ap. J. 116:1009.

Schmidt, B.P., Ap. J. 507 (1998) 46-63. 
A.I. Shylakhter, Nature 264, 340(1976).

J.K. Webb, V.V. Flambaum, C.W. Churchill, M.J. Drinkwater and J.D. Barrow, Phys. Rev. Lett. $82(1999) 884-887$.

I. Zlatev, L. Wang, P. Steinhardt, Phys. Rev. Lett. 82 (1999) 896-899. 

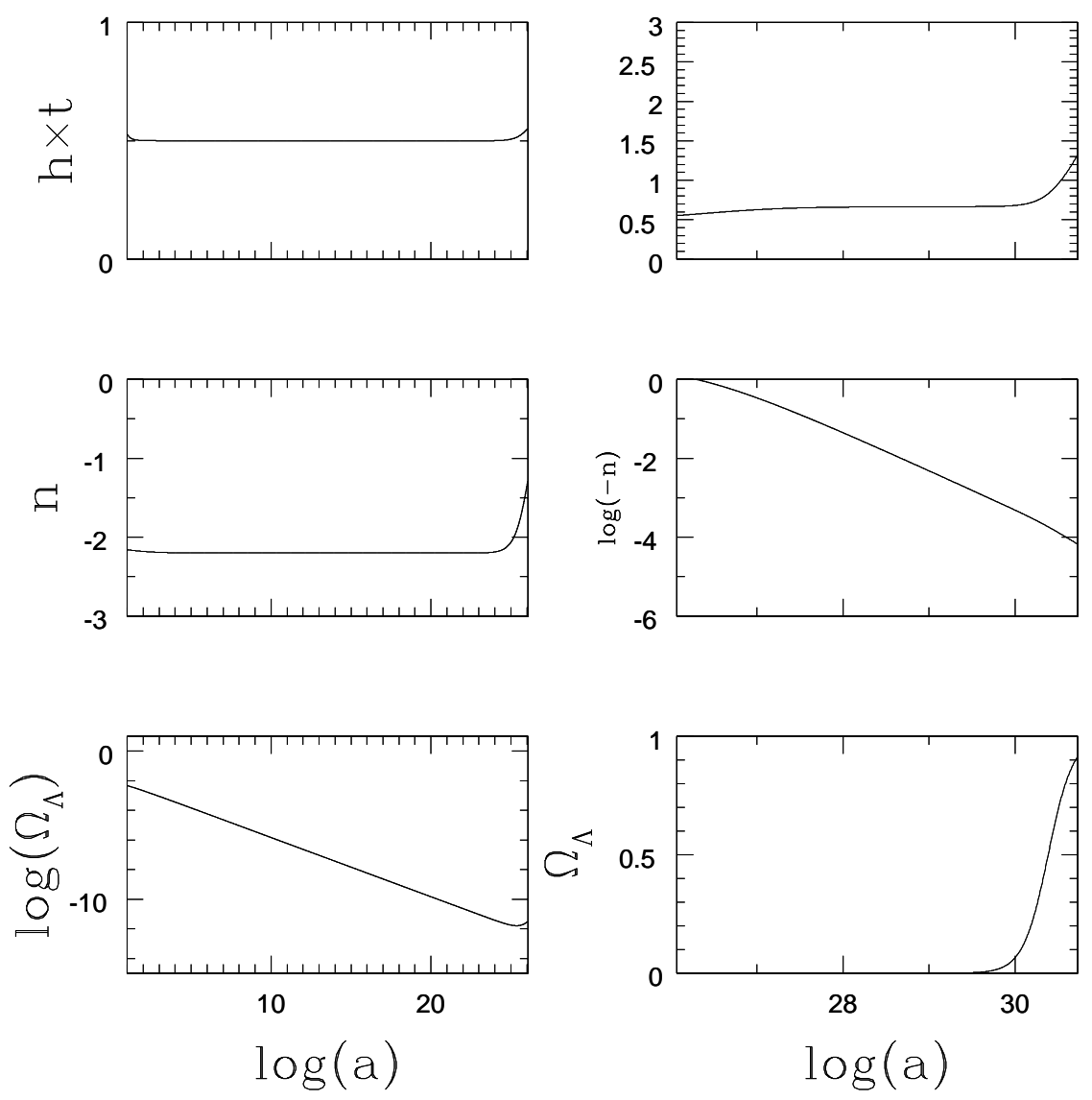

Fig. 1. - Evolution of $t \times h$ (where $h=\frac{\dot{a}}{a}$ ), $n=\frac{\dot{c} / c}{\dot{a} / a}$, and $\Omega_{\Lambda}$ in $\log$ or linear plots as appropriate. The panels on the left (right) describe the radiation (matter) dominated epoch. We have taken $\omega=-4.4$ (a modest $10 \%$ tuning over the critical value $\omega=-4$ ). In the radiation epoch $n=-2.2$, $\Omega_{\Lambda}$ slowly drops to zero, and the expansion factor has the usual dependence $a \propto t^{1 / 2}$. As the Universe enters the matter epoch $n$ starts dropping towards zero, $a \propto t^{2 / 3}$, and then $\Omega_{\Lambda}$ starts to increase. Eventually $n$ is of order $10^{-5}$ and $\Omega_{\Lambda}$ is of order 1 . This type of behaviour occurs for a large, non finely tuned, region of couplings $\omega$ and initial conditions. 